\title{
Fatores culturais e ambientais associados à evolução no conhecimento de herbáceas com potencial terapêutico
}

\author{
Josiene Maria Falcão Fraga dos Santos ${ }^{1}$; Elcida de Lima Araújo ${ }^{2}$; Ulysses Paulino de Albuquerque \\ ${ }^{1}$ Professora adjunta da Universidade Estadual de Alagoas, Núcleo de Biologia, Palmeira dos Índios, Alagoas; \\ ${ }^{2}$ Professora titular da Universidade Federal Rural de Pernambuco, Departamento de Biologia, Recife, Pernambuco; \\ ${ }^{3}$ Professor titular da Universidade Federal de Pernambuco, Departamento de Botânica, Recife, Pernambuco. \\ e-mail: josiene.falcao@uneal.edu.br
}

\section{Resumo}

As herbáceas representam grande valor para as populações humanas desde a antiguidade, fornecendo diversos recursos necessários à sua subsistência, sendo o uso medicinal o que recebe maior destaque entre as categorias citadas. Apesar disso, existem poucos estudos científicos que considerem o seu potencial de uso terapêutico e fatores como a história local das populações e questões ecológicas como a variação temporal (na disponibilidade do recurso) e variação espacial (em função de níveis de antropização). Diante do baixo número de estudos etnobotânicos específicos com herbáceas, torna-se arriscado concluir, nesse momento, um padrão geral das influências que direcionam o conhecimento e uso das mesmas. Entretanto, dentro dessa perspectiva, é possível mostrar uma tendência geral sobre os principais fatores atenuantes que regem o conhecimento e uso das herbáceas e que muitas vezes estão mascarados por contextos mais amplos, como por exemplo: fatores socioeconômicos, culturais, ecológicos e biológicos. No geral, esses elementos atuam em conjunto, para determinar qual o perfil dos usuários que dependem das herbáceas como recurso terapêutico e assim, aumentando o repertório das farmacopeias conhecidas localmente.

Palavras-chave: Áreas antropizadas, ervas medicinais, relação homem-natureza.

\section{Introdução}

O conhecimento sobre as plantas sempre tem acompanhado a evolução do homem através dos tempos. As primeiras civilizações perceberam de imediato a existência das plantas comestíveis e o poder daquelas com potencial curativo, embora algumas tivessem uma toxicidade não compatível com o organismo humano (Di Stasi et al. 1996). Inicialmente, essa descoberta relacionada à cura de doenças, era parte de um mundo empírico e toda essa informação foi transmitida às gerações posteriores (Moreira et al. 2002).

As primeiras informações documentadas sobre as ervas relatam sua importância nos cerimoniais de magia e medicina há cerca de 3.000 anos a.C. na Babilônia. Esses documentos traziam ilustrações contendo tratamentos médicos. Durante os 1000 anos subsequentes, culturas paralelas na China, Assíria, Egito e Índia, desenvolveram registros escritos de ervas medicinais onde existem antigos registros ocidentais que descrevem uma mistura de utilizações medicinais e mágicas para as plantas, com receitas médicas e anotações sobre a utilização aromática e cosmética das ervas (Botelho 2019).

No antigo Egito, as plantas condimentares eram usadas de muitas formas, deixando-as até mesmo nas tumbas dos faraós e personalidades importantes, para que estes fizessem viagem segura aos outros planos da existência, segundo suas crenças. São comuns citações dos papiros relatando a adoração que o povo tinha pelas plantas. $\mathrm{O}$ mais famoso deles é o Papiro Ebers, datado de 1550 a.C., que contém centenas de fórmulas e remédios populares usados na época (Carvalho e Almança 2003).

No Brasil, possivelmente, a utilização das plantas, não só como alimento, mas também como fonte terapêutica começou desde que os primeiros habitantes chegaram ao país, há cerca de $12 \mathrm{mil}$ 
anos, dando origem aos primeiros índios amazônicos, dos quais derivaram as principais tribos indígenas. Pouco, no entanto, se conhece sobre esse período, além das pinturas rupestres. As informações mais concretas que se tem conhecimento sobre os usos das herbáceas no Brasil foram registradas durante o período da colonização portuguesa, através do padre José de Anchieta. Ele detalhou informações sobre as plantas comestíveis como o feijão, o trigo, a cevada, o milho, o grão de bico, a lentilha, o cará, o palmito e a mandioca, que era o principal alimento dos índios e ainda sobre as plantas medicinais do Brasil em suas cartas ao superior geral da companhia de Jesus (Gordon 1996).

Muitos anos já se passaram e as plantas continuam sendo utilizadas com diversas finalidades que agora são registradas e publicadas no âmbito científico. Não apenas no Brasil, mas em várias regiões do mundo, o conhecimento e o uso das herbáceas, parecem ser impulsionados não apenas pela tradição histórico-cultural de cada povo, mas também pelas características ambientais de cada região (Albuquerque et al. 2005). A respeito disso, sabe-se que a disponibilidade temporal de plantas em regiões semiáridas pode interferir no uso de recursos vegetais, pois no período seco a densidade de espécies com hábito herbáceo diminui consideravelmente (Albuquerque et al. 2005, Reis et al. 2006), restando como recursos úteis nesse período, os indivíduos lenhosos da vegetação. Em áreas antropizadas da caatinga, o comportamento das espécies herbáceas é o mesmo de áreas preservadas no que se refere às flutuações de densidade entre as sazonalidades climáticas. No entanto, em áreas antropizadas, a composição florística sofre alterações devido a uma maior oferta de espécies ruderais e invasoras, predominantemente herbáceas (Albuquerque e Andrade 2002a, Albuquerque et al. 2007, Santos et al. 2009).

Albuquerque et al. (2005) enfatizam que a disponibilidade desses recursos e atendimento das necessidades do grupo obedece a fatores temporais, pois a distribuição de coleta no tempo mostrou-se dependente da disponibilidade sazonal dos recursos. Esse fato se deve principalmente a presença abundante das ervas, que concentram o seu ciclo de vida durante o período chuvoso. Dessa forma, a riqueza biológica da caatinga, que é marcada pela sazonalidade, merece ser melhor estudada levando em consideração tanto os parâmetros socioculturais, quanto os ecológicos, como o regime de chuva e o nível de antropização, por exemplo.

O cenário acima permite visualizar que a variação temporal existente na vegetação da caatinga, aliada com a dinâmica ecológica das plantas, influencia a dinâmica da relação homemrecurso vegetal. Contudo, é possível que a dinâmica da relação homem-recurso também afete a dinâmica ecológica das plantas, sobretudo se houver preferências por áreas de coleta (preservada ou antropizada).

Em várias partes do planeta, área de vegetação nativa tem sido transformada, em áreas de pastagens, terras agricultáveis e outros tipos de uso do solo, de forma que as paisagens se apresentam alteradas pelas atividades antrópicas (Ekpe 2002, Casteletti et al. 2003). Esse cenário de antropização tem aumentado em países da África, como em Gana, por exemplo, onde se estima que entre 60 e $80 \%$ das florestas originais já tenham sido perdidas (Fairhead e Leach 1998). Esse processo de conversão de áreas de vegetação nativa em florestas antrópicas continua acelerado, pois entre os anos 1990 e 2000, Gana perdeu $25 \%$ de sua cobertura florestal, equivalente a cerca de 1,7\% ao ano (WDI 2005). Em 2001, Gana já possuía uma população com cerca de 22,4 milhões de pessoas e esse crescimento populacional resultou na sobre-exploração dos recursos naturais (Otsuka 2001).

Essa realidade também ocorre na América do Sul, sobretudo na região semiárida do Brasil, a qual abriga uma das populações mais numerosas dentre as regiões semiáridas do mundo, com cerca de 28 milhões de habitantes. Desse total, $38 \%$ estão concentradas nas zonas rurais (Castro 2018). Na Caatinga, vegetação característica dessa região, as pressões antropogênicas têm sido percebidas mais fortemente nos últimos anos, e podem ser resumidas pela habitação humana e exploração da vegetação presente, principalmente por práticas agropecuárias (Albuquerque e Andrade 2002b, Sampaio et al. 2002, Araújo et al. 2007). Como resultado dessa exploração na caatinga, observa-se um mosaico vegetacional em diferentes estágios de regeneração (Pereira et al. 2003).

Embora tenhamos visto que o processo de antropização das florestas tropicais é crescente, essas áreas também são detentoras de uma diversidade biológica que precisa ser considerada, uma vez que algumas espécies ocorrem exclusivamente em áreas modificadas pelo homem (Albuquerque et al. 2005, 
Hanazaki et al. 2006). Tendo em vista esta exclusividade de espécies (a maioria herbácea) nas áreas antropizadas, alguns estudos sugerem que estas áreas atuam como fontes potenciais de recursos para o homem (Voeks 1996, Caniago e Siebert 1998, Voeks e Nyawa 2001, Ladio et al. 2007, Santos et al. 2014).

Trabalhos etnobotânicos em áreas perturbadas, que demostram existir uma variação na disponibilidade dos recursos vegetais, quando comparada a áreas preservadas, já foram realizados em ambientes de floresta tropical úmida no Brasil (Mata Atlântica). Como exemplo disso, tem o trabalho realizado por Voeks (1996), que investigando as preferências dos curandeiros quanto à busca de recursos em florestas tropicais preservada e antropizada, afirma que essa última constitui uma fonte importante para obtenção de plantas úteis e se caracterizam por terem uma ampla variedade com potencial de uso medicinal. Segundo o autor, a população local prefere usar medicamentos extraídos das plantas de áreas antropizadas. Ele atribui essa preferência a fatores como mudanças culturais, que afetaram o conhecimento sobre o uso dos recursos destinados para fins medicinais em áreas de vegetação preservada. Também podem explicar essa preferência, a disponibilidade relativa elevada e o potencial inerente das espécies de áreas perturbadas, pois o estrato dominante é formado pelas herbáceas e esse grupo pode representar uma grande fonte de compostos bioativos.

Hanazaki et al. (2006) observaram quatro áreas de um fragmento de floresta tropical úmida, em diferentes estágios sucessionais. A primeira área foi considerada preservada, sem histórico de uso conhecido, a segunda foi formada por uma área de cultivo em atividade, a terceira por uma área em estágio sucessional avançado (50 anos de preservação) e a quarta é constituída por uma área recentemente perturbada (bordas de estradas $\mathrm{e}$ trilhas, áreas de plantio abandonados recentemente). Os autores registraram a mesma tendência que vem sendo apontada por outros autores nas florestas tropicais. As plantas medicinais foram obtidas principalmente da área de cultivo e da área recentemente perturbada, enquanto que a maioria das plantas utilizadas com fins madeireiros foi obtida da área preservada. O grupo de espécies utilizadas para fins medicinais é formado principalmente por plantas herbáceas da área cultivada. Entre as lenhosas encontradas neste estudo, poucas são citadas para usos medicinais, pois a importância da maioria dessas espécies está no uso da madeira.

Numa Floresta Equatorial Montana da Ásia, localizada no leste da Indonésia - Kalimantam, Caniago e Siebert (1998) compararam o conhecimento e a diversidade de plantas com potenciais de usos medicinais em áreas de vegetação com diferentes níveis de preservação. Os autores observaram que a maior parte da comunidade tem mais conhecimento sobre plantas medicinais encontradas no interior da floresta em processo inicial de sucessão secundária do que nas florestas primárias. Possivelmente isso se deve a maior diversidade de espécies na floresta em sucessão secundária, relatado pelos autores. Apesar da diversidade elevada e do conhecimento concentrado nesse tipo de floresta, foi constatado que a floresta primária é detentora de um considerável número de espécies exclusivas, consideradas importantes no tratamento de doenças raras pela comunidade estudada.

No mesmo contexto de vegetação úmida, porém localizada na América do Norte, em duas comunidades no México (Zapotecas e Mixe), Frei et al. (2000) obtiveram resultados que corroboram com a ideia de que áreas de vegetação perturbada são as fontes mais importantes de obtenção de plantas medicinais. Nas comunidades estudadas foram constatados que as plantas medicinais correspondem a uma parte essencial na cura das doenças tratadas localmente. Além disso, esse estudo analisou as estratégias etnoecológicas empregadas pelos grupos indígenas na busca de plantas medicinais. Assim a classificação indígena do ambiente é notadamente diferente da classificação ocidental e distingue seis zonas antropogênicas principais ou tipos de uso da terra. A maioria das espécies de importância etnomédica se cultiva nos quintais ao redor das casas ou é coletada na beira da estrada. Essas zonas, por exemplo, contribuem com $31,8 \%$ e $26,2 \%$ de todas as plantas medicinais dos Mixe e Zapotecas, respectivamente. Estes dados etnobotânicos sobre o uso indígena da terra indicam que os tipos de vegetação antropogênicas é muito importante para obtenção de plantas medicinais. No entanto, apenas $15,6 \%$ e $19,6 \%$ das plantas usadas pelas comunidades Mixe e Zapotecas, respectivamente, são provenientes da vegetação mais preservada. Em contraposição, On et al. (2001) afirmam que as plantas usadas como medicinais são 
preferencialmente encontradas em áreas de vegetação secundária mais fechada, e em locais com altitude maior.

Apesar de Blanckaert et al. (2007) não inferirem sobre a preferência por área de coleta de recursos herbáceos, na vegetação de clima semiárido quente do México, foi investigado a influência dessas áreas antrópicas sobre o conhecimento dos moradores locais. Cerca de $90 \%$ das espécies daninhas tinham uma citação de uso e a categoria mais expressiva informada pela comunidade, considerando o grupo herbáceo, foi a forrageira, seguida pelo uso medicinal. No semiárido brasileiro, Santos et al. (2009), registraram tendência semelhante com relação à importância das categorias de uso citadas. Os autores, investigando a composição, diversidade e uso de uma área antropogênica da caatinga, verificaram que as espécies herbáceas são utilizadas, predominantemente, como espécies forrageiras, ocorrendo também um amplo repertório de plantas utilizadas como medicinais. Sobre a preferência por área de coleta de recursos herbáceos em floresta seca no Brasil, até o momento não há um padrão definido, devido a carência de estudos dessa natureza. No entanto, já existe uma tendência sendo apontadas nessa questão. Albuquerque et al. (2005), por exemplo, constataram que áreas perturbadas oferecem elevada disponibilidade de plantas com potencial medicinal, mas apesar disso, as áreas antropizadas não retêm a preferência das pessoas para coleta. A comunidade estudada conhece as plantas medicinais da área perturbada, que em sua maioria são herbáceas de ciclo de vida curto, mas preferem utilizar as espécies da área preservada, mesmo que seja necessário um gasto de energia maior na busca para obter o produto desejado. Esse comportamento difere da tendência registrada para as florestas tropicais úmidas, podendo está relacionado à permanência dos valores culturais e dos conhecimentos básicos sobre as espécies presentes no interior da área preservada e, principalmente a disponibilidade temporal dos recursos, por se tratar de uma floresta estacional seca.

De maneira geral, a literatura etnobotânica já vem apontando que existe uma diferença quanto à seleção do local onde será extraído o recurso vegetal em muitas florestas tropicais. Todavia as diferenças florísticas por si só não explicam a preferência da comunidade por um determinado sítio ecológico.
Alguns trabalhos realizados em florestas tropicais úmidas mostraram que as pessoas preferem os recursos provenientes das áreas antropizadas (Voeks 1996, Hanazaki et al. 2006). Já em florestas tropicais secas, incluindo os de regiões frias, os trabalhos apontam uma preferência por áreas preservadas (On et al. 2001, Ladio e Lozada 2004, Albuquerque et al. 2005). No entanto, alguns trabalhos que discutem essa temática não fazem uma separação nítida entre o conhecimento e o uso, pois se sabe que quando uma espécie é citada numa entrevista, nem sempre isso quer dizer que a mesma seja realmente utilizada, já que ela pode estar compondo apenas o conhecimento do informante sobre plantas potencialmente úteis.

Essa divergência quanto ao comportamento de grupos humanos na seleção do local para extração de plantas, precisa ser melhor investigada, sugerindo que estudos devem ser concentrados em comunidades que habitam regiões semiáridas para testar se esse comportamento é isolado ou reflete um padrão de comportamento das pessoas que habitam nesses ecossistemas.

\section{Conclusão}

O conhecimento sobre plantas herbáceas com potencial de uso no tratamento ou cura de doenças é bastante vasto e dinâmico, pois podem sofrer com interferências culturais e também ambientais. Logo, entender os mecanismos associados diretamente a dinâmica no conhecimento de plantas com potencial terapêutico tem sido um desafio pois ao mesmo tempo em que podem contribuir na manutenção, podem também explicar a erosão do conhecimento dentro de uma comunidade tradicional. Enquanto o mundo se torna cada vez mais interligado, o potencial para a assimilação de uma cultura global dominante e a necessidade de conservação (incluindo o conhecimento) biocultural se tornam mais aparentes. Na expansão da população humana, a agricultura moderna, o crescimento econômico, o desrespeito com as práticas tradicionais são apenas algumas das ameaças ao conhecimento sobre o ambiente natural. Nesse contexto, o grande desafio dos pesquisadores é encontrar maneiras de ajudar as comunidades a conservar a identidade cultural e o conhecimento tradicional sem comprometer a capacidade de compreensão global e comunicação. Para fazer isso, temos que começar por uma melhor compreensão de culturas e identificar com maior 
precisão os fatores que estão relacionados a esse conhecimento.

\section{Referências}

1. Sousa MR, Albuquerque UP, Andrade LHC, Caballero J (2005). Structure and floristics of homegardens in Northeastern Brazil. Journal of Arid Environments 62:491-506.

2. Albuquerque UP, Andrade LHC (2002a). Conhecimento botânico tradicional e conservação em uma área de caatinga no estado de Pernambuco, nordeste do brasil. Acta Botanica Brasilica 16:273285.

3. Albuquerque UP, Andrade LHC (2002b). Uso de recursos vegetais da caatinga: o caso do agreste do Estado de Pernambuco (Nordeste do Brasil). Interciencia 27:336-346.

4. Albuquerque UP, Medeiros PM, Almeida ALS, Monteiro JM, Lins Neto EMF, Melo JG, Santos JP (2007). Medicinal plants of the caatinga (semi-arid) vegetation of NE Brazil: a quantitative approach. Journal of Ethnopharmacology 114:325-354.

5. Araújo EL, Castro CC, Albuquerque UP (2007). Dynamics of Brazilian Caatinga - A review concerning the plants, environment and people. Functional Ecosystems and Communities 1:15-28.

6. Blanckaert I, Vancraeynest K, Swennen RL, Espinosa García FJ, Piñero D, Lira-Saade R (2007). Non-crop resources and production of Mexico. Agriculture, Ecossystems and Environment 119:3948.

7. Botelho João Bosco. História da Medicina. www.historiadamedicina.med.br (Acesso no dia 05/06/2019).

8. Caniago I, Siebert ST (1998). Medicinal Plant Ecology Knowledge and Conservation In Kalimantan Indonesia. Economic Botany 52:229250.

9. Carvalho JCT, Almança CCJ (2003) Formulário de prescrição fitoterápica. Editora Atheneu, São Paulo.

10. Castelletti, C, Tabarelli M, Silva JMC (2003) "Quanto ainda resta da Caatinga? Uma estimativa preliminar," In: IR Leal, M Tabarelli, JMC Silva (Org.). Ecologia e Conservacão da Caatinga. Editora Universitária/ Universidade Federal de Pernambuco, Recife, Brasil.

11. Castro R. Acessado em 26/09/2018. Reservas privadas do bioma caatinga. http://www.sbpcnet.org.br/livro/57ra/programas/CO NF_SIMP/textos/rodrigocastro.htm.

12. Di Stasi LC, Brito ARMS, Bacchi EM, Ming LC, Furlan MR, Savastano MAP, Amorozo MC, Reis MS, Ferri PH (1996) "Plantas medicinais: arte e ciência, um guia de estudo interdisciplinar", Editora da Universidade Estadual Paulista, São Paulo.

13. Ekpe P (2002) Forest loss in Ghana and its impact on access to wild medicinal plants. In: H. Gillett (Org.). Conservation and Sustainable Use of Medicinal Plants in Ghana, Conservation report UNEP-WCMC.

14. Fairhead J, Leach M (1998) Reframing Deforestation: Global analyses and local realities in West Africa. Global Environmental Change Programme. Routledge. New York.

15. Frei B, Sticher O, Heinrich M (2000) Zapotec and mixe use of tropical habitats for securing medicinal plants in Mexico. Economic Botany 54:3-81.

16. Gordon R (1996) A Assustadora história da medicina. Rio de janeiro. Ed. Ouro.

17. Hanazaki N, Souza VC, Rodrigues RR (2006) Ethnobotany of rural people from the boundaries of Carlos Botelho State Park, São Paulo State, Brazil Acta Botanica Brasilica 20:899-909.

18. Hanazaki N, Souza VC, Rodrigues RR (2006) Ethnobotany of rural people from the boundaries of Carlos Botelho State Park, São Paulo State, Brazil Acta Botanica Brasilica 20:899-909.

19. Ladio AH, Lozada M (2004) Patterns of use and knowledge of wild edible plants in distinct ecological environments: a case study of a Mapuche community from northwestern Patagonia. Biodiversity and Conservation 13:1153-1173.

20. Ladio A, Lozada M, Weigandt M (2007) Comparison of traditional wild plant knowledge between aboriginal communities inhabiting arid and forest environments in Patagonia, Argentina Journal of Arid Environments 69: 695-715.

21. Moreira RCT, Bomfim LC, Costa RCS, ROCHA EA (2002) Abordagem Etnobotânica acerca do Uso de Plantas Medicinais na Vila Cachoeira, Ilhéus, Bahia, Brasil Acta Farm. Bonaerense 21: 205-11.

22. On TV, Quyen D, Bich LD, Jones B, Wunder JB, Smith-Russel J (2001) A survey of medicinal plants in BaVi National Park, Vietnam: methodology and implications for conservation and sustainable use. Biological Conservation 97:295-304.

23. Otsuka A (2001) Population Pressure, Land Tenure, and Natural Resource Management. Tokyo, Asian Development Bank Institute: 27.

24. Pereira IM, Andrade LA, Sampaio EVSB, Barbosa MRV (2003) Use-history effects on structure and flora of Caatinga. Biotropica 35:154-165.

25. Reis AM, Araújo EL, Ferraz EM, Moura AN (2006) Inter-annual variations in the floristic and population structure of an herbaceous community of "Caatinga" vegetation in Pernambuco, Brazil. Revista Brasileira de Botânica 29:497-508.

26. Sampaio EVSB, Giulietti AM, Virgínio J, GamarraRojas CFL (2002) Vegetação e flora da caatinga. 
Associação Plantas do Nordeste - APNE. Centro Nordestino de Informação sobre Plantas, Recife.

27. Santos LL, Nascimento ALB, Vieira FJ, Silva VA, Voeks R, Albuquerque UP (2014) The Cultural Value of Invasive Species: A Case Study from Semi-Arid Northeastern Brazil. Economic Botany 68:283-300.

28. Santos LL, Ramos MA, Izídio SI, Sales MF, Albuquerque UP (2009) Caatinga Ethnobotany: Anthropogenic Landscape Modification and Useful Species in Brazil's Semi-Arid Northeast. Economic Botany 63:363-374.

29. Voeks RA (1996) Tropical forest healers and habitat preference. Economic Botany 50:381-400.

30. Voeks RA, Nyawa S (2001) Healing flora of the Brunei Dusun. Borneo Research Bulletin 32:178195.

31. WDI (World Development Indicators) 2005. Ghana: Statistics (1960-2005). 